\title{
Mutagenic specificity of endogenously generated abasic sites in Saccharomyces cerevisiae chromosomal DNA
}

\author{
Paul Auerbach*, Richard A. O. Bennett*, Elisabeth A. Bailey*, Hans E. Krokan ${ }^{\dagger}$, and Bruce Demple** \\ *Department of Genetics and Complex Diseases, Harvard School of Public Health, Boston, MA 02115; and ${ }^{\dagger}$ Department of Cancer Research and Molecular \\ Medicine, Norwegian University of Science and Technology, N-7489 Trondheim, Norway
}

Edited by Aziz Sancar, University of North Carolina, Chapel Hill, NC, and approved October 16, 2005 (received for review June 3, 2005)

\begin{abstract}
Abasic [apurinic/apyrimidinic (AP)] sites are common, noncoding DNA lesions. Despite extensive investigation, the mutational pattern they provoke in eukaryotic cells remains unresolved. We constructed Saccharomyces cerevisiae strains in which chromosomal AP sites were generated during normal cell growth by altered human uracil-DNA glycosylases that remove undamaged cytosines or thymines. The mutation target was the URA3 gene inserted near the ARS309 origin to allow defined replication polarity. Expression of the altered glycosylases caused a 7- to 18-fold mutator effect in AP endonuclease-deficient ( $\Delta a p n 1)$ yeast, which depended highly on the known translesion synthesis enzymes Rev1 and DNA polymerase $\zeta$. For the C-glycosylase, GC >CG transversions were the predominant mutations, followed by GC > AT transitions. AT>CG transversions predominated for the T-glycosylase. These results support a major role for Rev1-dependent dCMP insertion across from AP sites and a lesser role for dAMP insertion. Unexpectedly, there was also a significant proportion of dTMP insertions that suggest another mutational pathway at AP sites. Although replication polarity did not strongly influence mutagenesis at AP sites, for certain mutation types, there was a surprisingly strong difference between the transcribed and nontranscribed strands of URA3. The basis for this strand discrimination requires further exploration.
\end{abstract}

A basic [apurinic/apyrimidinic (AP)] sites in DNA are very common lesions that arise directly from spontaneous base hydrolysis and free-radical reactions or indirectly through base damage caused by diverse endogenous and exogenous agents (1). The mutagenic effects of unrepaired AP sites result from DNA synthesis on these damaged templates (2). The normal replicative DNA polymerases cannot readily copy past AP sites (2), which, instead, require a specialized class of enzymes, the translesion synthesis (TLS) enzymes (for a review, see ref. 3). The presumptive evolutionary advantage of TLS enzymes is to allow cell survival of otherwise lethal DNA damage. Because AP sites lack the biochemical information required to identify the correct base, TLS enzymes may insert a nucleotide that results in a mutation.

The known TLS-related proteins in Saccharomyces cerevisiae, Rev1, Rev3, Rev7, Pol32, and Rad30, have been characterized genetically and biochemically. Yeast Rev1 acts in vitro as a deoxycytidyl transferase, inserting dCMP opposite lesions (4). Rev1 also has a modulatory role for other TLS enzymes (5). Pol32 is a nonessential subunit of the major yeast replicative DNA polymerase $\delta$ that promotes insertion of, predominantly, dAMP opposite AP sites in vitro (6). These insertions are extended by the postmismatch DNA polymerase $\zeta$, a heterodimer of Rev3 and Rev7 proteins (5). Rad30 encodes DNA polymerase $\eta$, which, in vitro, inserts mainly dGMP or dAMP opposite AP sites (7), although recent in vivo studies do not support such a role $(8,9)$. After extension, synthesis switches back to normal DNA-replication enzymes $(10,11)$.

In a study using the yeast SUP4-o system, the most dramatic mutational effect of Apn1-deficiency was the generation of AT $>$ CG transversions (12). These mutations depended partly on the Mag1 3-methyladenine-DNA glycosylase, which led to the suggestion that such transversions might represent preferential dGMP insertion opposite the AP site. This interpretation rested on a narrow view of Mag1 specificity, and later work showed preferential insertion of dCMP during DNA synthesis past AP sites on plasmids transformed into yeast (13). Yeast transformation with AP-site-containing oligonucleotides (14) also suggested a "C rule" for insertion by TLS enzymes. A recent study indicates that spontaneous AP sites in $S$. cerevisiae are due mostly to excision of uracil incorporated opposite adenine (15). That observation may account for the AT>CG transversions found with SUP4-o (12), although it does not explain the partial MAG1 dependence of those mutations.

Examination of mutagenesis in cells treated with DNA-damaging agents leads to somewhat different conclusions. Mutagenesis in yeast exposed to methyl methanesulfonate (which produces numerous AP sites) suggested preferential insertion of dAMP, consistent with an "A rule" for repair of abasic sites (5). A significant limitation of this interpretation, however, is that most of the observed mutations involved GC base pairs, where dCMP incorporation opposite a G-derived AP site would restore the wild-type sequence. Thus, uncertainties about the identity of the mutagenic AP sites attend both traditional studies of spontaneous mutation and studies of mutation induced by DNA-damaging agents.

Although the approach of using plasmids or oligonucleotides with known AP sites or glycosylase targets has been informative, it is uncertain whether DNA replication after introduction into the cells accurately reflects the processes acting on chromosomal genes. We have now overcome these limitations by employing modified versions of human uracil-DNA glycosylase (UDG) that remove either normal cytosines or normal thymines from yeast nuclear DNA (16), coupled to a high-throughput approach that allows all of the main classes of mutations to be identified at statistically significant levels.

\section{Materials and Methods}

Strains and Plasmids. Most of the yeast strains used here were derived from strain BGY111 (MATa, his3- $\Delta$, leu2, ura3-52, trp1$289 a$, can1, and $G A L^{+}$) (17). BGY111 was modified by transformation with pNKY1009, resulting in a trp1- $\Delta 1:$ his $G$ genotype (18), to generate strain LBY2. LBY2 strains carrying a deletion of $A P N 1$ were constructed by transformation with pSCP19A (19), carrying an apn1- $\Delta 1:: H I S 3$ module, to create the strain LBY2a. Strains

Conflict of interest statement: No conflicts declared.

This paper was submitted directly (Track II) to the PNAS office.

Abbreviations: AP, Abasic (apurinic/apyrimidinic); CDG, cytosine-DNA glycosylase; CM-leu, leucine-deficient complete minimal medium; ODG, oxanine-DNA glycosylase; TDG, thymine-DNA-glycosylase; TLS, translesion synthesis; UDG, uracil-DNA-glycosylase; 5-FOA, 5-fluoroorotic acid.

\#To whom correspondence should be addressed at: Department of Genetics and Complex Diseases, Harvard School of Public Health, 655 Huntington Avenue, Building I, Room 509, Boston, MA 02115. E-mail: bdemple@hsph.harvard.edu.

(C) 2005 by The National Academy of Sciences of the USA 


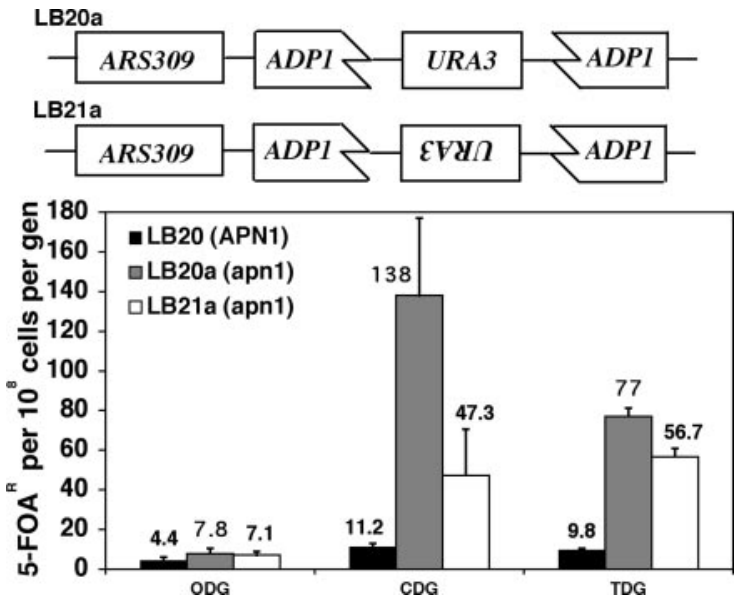

Fig. 1. Mutation rates in yeast expressing the CDG and TDG glycosylases. The upper part of the figure shows the structure of the URA3 mutation targets inserted at the ADP1 locus in strains LB20a and LB21a. Mutation rates to ura3 were estimated by selecting for resistance to 5-FOA, as described in the text.

LBY2 and LBY2a were further modified by introducing URA3 as a disruption of the $A D P 1$ gene. The nonessential $A D P 1$ gene (20) is located on chromosome III $\approx 1.7 \mathrm{~kb}$ from the $5^{\prime}$ end of the early firing ARS309 replication origin (21) (Fig. 1). Control experiments showed that this disruption did not affect the spontaneous mutation rate (to $\mathrm{Can}^{\mathrm{R}}$; data not shown). Construction of the DNA used for the disruption of the $A D P 1$ gene with $U R A 3$ inserted in both orientations is provided in Supporting Text, which is published as supporting information on the PNAS web site.

These manipulations yielded strains LB20 (APN1) and LB20a (apn1- $\triangle 1:: H I S 3$ ) with $U R A 3$ transcriptional orientation as for $A D P 1$ and LB21 (APN1) and LB21a (apn1- $11:: H I S 3)$ with URA3 transcriptional orientation opposite $A D P 1$ (Fig. 1). The ADP1::URA3 disruption had no detectable effect on the cell growth rate or cellular sensitivity to various mutagens (UV light, methyl methanesulfonate, and hydrogen peroxide).

Strain FY69 (APN1, URA3) was provided by Fred Winston of the Department of Genetics at Harvard Medical School (Boston).

Construction of rev1 and rev7 Knockout Strains. Knockout strains were constructed by using the protocols of the Saccharomyces Genome Deletion Project (http://sequence-www.stanford.edu/ group/yeast_deletion_project). BY4741 yeast strains with the REV1 or REV7 locus replaced with the G418 (geneticin)-resistance cassette kanMX4 were purchased from American Type Culture Collection, and the cassettes with flanking genomic sequences were amplified by PCR. The resulting PCR fragments were used to transform strain LB20a. After selection by G418 resistance, gene replacement was confirmed by PCR. The transformants were further screened for increased sensitivity to killing by 254-nm UV light (22). The rev1::kanMX4 strain was designated PAY01a, and the rev7::kanMX4 strain was designated PAY07a.

Construction of Glycosylase Expression Vector. Derivatives of the human uracil glycosylase gene encoding mutant enzymes that excise normal thymines or cytosines from DNA are described in ref. 16. Excision of the glycosylase genes from the original vectors and ligation into plasmid pYES2 is detailed in Supporting Text. The plasmids produced were pODG, the empty vector control; pCDG, encoding the cytosine-excising enzyme; and pTDG, encoding the thymine-excising enzyme (16).

Glycosylase expression was controlled by the GAL1 promoter. For routine growth, strains bearing the glycosylase expression plasmids were cultured in leucine-deficient complete minimal medium (CM-leu) (23) with glucose to maintain the plasmid and inhibit glycosylase expression. For glycosylase expression, strains were transferred to CM-leu with raffinose to clear the cells of glucose and then to CM-leu containing galactose (cells from the raffinose cultures seeded at $\approx 3,000$ cells per $\mathrm{ml}$ ) and the cultures incubated as described below in Identification and Sequencing of Ura3- Mutants).

Western Blot Analysis. Liquid cultures for LB20a and LB21a strains containing pODG, pCDG, or pTDG were grown to saturation during a 2-3 day incubation in CM-leu containing glucose, raffinose, or galactose. Cultures containing galactose were seeded from cells grown in raffinose, as described above. Protein extracts of these cultures were prepared under denaturing conditions (24). Western blot analysis was performed as described in the Fig. 4, which is published as supporting information on the PNAS web site.

Identification and Sequencing of Ura3- Mutants. Stains carrying putative mutations in $U R A 3$ were selected on medium containing 5-fluoro-orotic acid (5-FOA, Research Products International, Mt. Prospect, IL) (25). The 5-FOA-resistant colonies were then restreaked onto plates containing complete minimal medium lacking uracil (CM-ura) to confirm the expected uracil auxotrophy. The 5-FOA-resistant colonies unable to grow on CM-ura plates were inoculated into 1-ml CM-leu plus glucose. After the cultures reached saturation $(\approx 2$ days $)$, the cells were collected and genomic DNA extracted by using a kit (Epicentre, Madison, WI), and the inserted URA3 locus was amplified by PCR. The PCR conditions were an initial denaturation at $94^{\circ} \mathrm{C}$ for $5 \mathrm{~min} ; 35$ cycles of $94^{\circ} \mathrm{C}$ for $0.5 \mathrm{~min}, 56^{\circ} \mathrm{C}$ for $0.5 \mathrm{~min}, 72^{\circ} \mathrm{C}$ for $2.5 \mathrm{~min}$, and a final extension step at $72^{\circ} \mathrm{C}$ for $7 \mathrm{~min}$. Samples containing a PCR product with size corresponding to the full $U R A 3$ gene $(\approx 1.3 \mathrm{~kb})$ were then purified (Qiagen, Valencia, CA), sequenced, and the results catalogued. The DNA sequencing was performed at the Dana-Farber/Harvard Cancer Center High-Throughput DNA Sequencing Facility.

Determination of Mutation Rates. Mutation rates were determined by using the method of the median (26). Briefly, 20 5-ml cultures were first grown to saturation, as described above, in CM-leu plus galactose. Three samples from each culture tube were plated onto 5 -FOA plates and three diluted $(1: 10,000)$ samples onto yeast extract/bacto-peptone/dextrose (YPD) plates. The mean number of colonies on the YPD plates was divided into the corresponding total number of 5-FOA ${ }^{\mathrm{R}}$ colonies to give a mutation frequency for each culture. The median value of the 20 mutation frequencies was then used to estimate the mutation rate from a statistical table (26).

Categorization of Orientation Differences. Mutations were scored in relation to the $U R A 3$ coding sequence (nontranscribed strand), so that, in the case of cytosine-DNA-glycolsylase (CDG)-induced mutants, a substituted cytosine indicates that the mutation originated from a base removed in the nontranscribed strand, whereas a substituted guanine indicated that the mutation originated from a base removed in the transcribed strand. Thymine-DNAglycosylase (TDG)-induced mutants were scored analogously. As shown in Fig. 1 (Top), in the LB20 background, URA3 is expressed codirectionally with leading-strand DNA synthesis, with $U R A 3$ in the LB21 background having the opposite orientation.

Statistical Analyses. Differences in the frequencies of strand-specific base substitutions were analyzed for significance by a comparison of proportions or $\chi^{2}$ analysis. In each case, a $P$ value $<0.05$ indicated a significant difference and a rejection of the null hypothesis (that there were no strand-specific differences in base substitutions).

\section{Results}

Mutagenesis Driven by Mutant UDGs. The mutagenic effects of AP sites formed by the modified glycosylases (CDG and TDG) in $S$. cerevisiae were explored initially in three strain backgrounds: LB20 
Table 1. Dependence of mutator glycosylases on REV1 and REV7

Mutation rate for glycosylase vector, mutants per $10^{8}$ cells per generation

\begin{tabular}{llcc}
\cline { 2 - 4 } Strain & \multicolumn{1}{c}{ ODG } & \multicolumn{1}{c}{ CDG } & TDG \\
\hline LB20a & $7.8 \pm 2.5$ & $138 \pm 39$ & $77 \pm 4.2$ \\
PAY01a (rev1) & $0.6 \pm 0.59$ & $4.6 \pm 4.1$ & $1.8 \pm 2.2$ \\
PAY07a (rev7) & $4.2 \pm 3.9$ & $5.6 \pm 5.0$ & $7.1 \pm 1.3$ \\
\hline
\end{tabular}

$\left(A P N 1^{+}\right)$, LB20a (apn1- $\left.\Delta 1\right)$, and LB21a (apn1- $\left.\Delta 1\right)$, which represent the two replication orientations of the $U R A 3$ gene (Fig. 1). For the strains containing pODG, deletion of APN1 caused a modest increase in the mutation rate (Fig. 1), consistent with reports in refs. 12, 27, and 28. The additional deletion of the second yeast AP endonuclease gene (APN2/ETH1) further increased the spontaneous mutation rate $(29,30)$, but expression of the mutant glycosylases was lethal in a apn1 1 apn $2 \Delta$ strain (data not shown).

Expression of the CDG and TDG glycosylases increased the mutation rate in the APN1 (repair-proficient) background and in both apn1- $\Delta 1$ strains. In $A P N 1$ cells, the increase was modest, 2.5 fold for CDG and 2.2 fold for TDG (Fig. 1). In contrast, the mutagenic effect of the altered glycosylases was much greater in the apn 1- $\Delta 1$ strains. In LB20a, expression of CDG increased the mutation rate $\approx 18$-fold over the empty vector (pODG), whereas expression of TDG increased the mutation rate $\approx 10$-fold. In LB21a, expression of $\mathrm{CDG}$ increased the mutation rate $\approx 7$-fold and TDG by 8-fold relative to oxanine-DNA glycosylase (ODG) (Fig. 1). CDG and TDG are derived from human UDG, but a construct expressing the normal UDG sequence did not increase the mutation rate in apn $1-\Delta 1$ yeast (data not shown). Thus, the CDG and TDG effects were not likely because of interference with normal yeast processes by the human protein.

Because CDG and TDG are DNA $N$-glycosylases with no other known enzymatic activity (16), and because their mutator effects were much greater in an apn $1-\Delta 1$ background than in APN1 cells, the mutagenic effect of these enzymes is most likely due to the formation of AP sites. We attempted to use the aldehyde-specific probe assay (31) to measure the production of the "extra" AP sites due to these enzymes, but this approach was unsuccessful (data not shown). The assay suffers from a rather high background because of AP sites generated during DNA extraction and handling that obscure the contribution of the endogenous damage (32); indeed, even the lethal generation of AP sites by CDG and TDG in Escherichia coli did not produce a measurable signal in this assay (33). Combined with the observation that CDG or TDG expression under our conditions had only a small effect on the growth rate of apn $1-\Delta 1$ yeast strains (see Table 5 , which is published as supporting information on the PNAS web site), these considerations imply that the mutator-glycosylase-driven mutagenesis results from the generation of relatively low numbers of AP sites.

Requirement of Rev1 and Pol $\zeta$ for Mutagenesis. To test the hypothesis that the increased mutation rates were due to the activity of TLS enzymes, we constructed yeast strains deficient in Rev1 or Pol $\zeta$. Expression of the mutator glycosylases slowed the growth of strains PAY03a and PAY07a moderately and of PAY01a more dramatically (see Fig. 5, which is published as supporting information on the PNAS web site). Both TLS-deficient strains showed a marked decrease in CDG- or TDG-induced mutagenesis compared with strain LB20a (Table 1). In addition, the Rev1-deficient strain PAY01a had a spontaneous mutation rate (with the $\mathrm{pODG}$ vector) that was $\approx 13$-fold lower than that of LB20a. Compared with LB20a, the rate in PAY01a was $\approx 30$-fold lower when CDG was expressed and $\approx 40$-fold lower when TDG was expressed. In the Rev7deficient strain PAY07a, compared with LB20a, the spontaneous mutation rate was $\approx 2$-fold lower with $\mathrm{pODG}, \approx 25$-fold lower when CDG was expressed, and $\approx 10$-fold lower when $T D G$ was expressed.
Table 2. Overall URA3 mutation spectra

\begin{tabular}{|c|c|c|c|}
\hline \multirow[b]{2}{*}{ Mutations } & \multicolumn{3}{|c|}{ Vector } \\
\hline & ODG & CDG & TDG \\
\hline \multicolumn{4}{|l|}{ LB20a (apn1- $\Delta 1)$} \\
\hline Total analyzed & 232 & 375 & 419 \\
\hline Point mutations & $85(37)$ & $211(56)$ & $239(57)$ \\
\hline 290-bp deletions & $136(58)$ & 147 (39) & $157(38)$ \\
\hline Other & $11(5)$ & $17(5)$ & $23(5)$ \\
\hline \multicolumn{4}{|l|}{ LB21a $(a p n 1-\Delta 1)$} \\
\hline Total analyzed & 81 & 192 & 274 \\
\hline Point mutations & $44(54)$ & $91(48)$ & $142(53)$ \\
\hline 290-bp deletions & $29(35)$ & $85(44)$ & $100(36)$ \\
\hline Other & $8(10)$ & $16(8)$ & $32(12)$ \\
\hline \multicolumn{4}{|l|}{ LB20 (APN1) } \\
\hline Total analyzed & 0 & 89 & 90 \\
\hline Point mutations & $\mathrm{N} / \mathrm{A}$ & $41(46)$ & $44(49)$ \\
\hline 290-bp deletions & $\mathrm{N} / \mathrm{A}$ & $46(52)$ & $40(44)$ \\
\hline Other & $\mathrm{N} / \mathrm{A}$ & $2(2)$ & $6(7)$ \\
\hline
\end{tabular}

Data in parentheses are percentages. N/A, not applicable.

Thus, the mutation spectra reported here are dominated by processes that depend on Rev1 and Pol $\zeta$.

Mutational Specificity of AP Sites in S. cerevisiae. To examine the mutation spectrum for AP sites in yeast, we isolated cells with ura3 mutations, which confer both resistance to 5-FOA and a $\mathrm{Ura}^{-}$ phenotype (see Materials and Methods). As summarized in Table 2, three categories of mutations were catalogued in both the LB20a and LB21a backgrounds: predominantly single-base-pair substitutions, a specific 290-bp deletion, and a few other types, such as small deletions and insertions. In each case, single-base-pair substitutions accounted for about $40-60 \%$ of the total mutations, the 290-bp deletion accounted for $40-60 \%$, and the "other" category accounted for $5-10 \%$ of the ura3 mutants. This general distribution of mutation types was consistent between the two orientations of the URA3 mutation target (Table 2). The 290-bp deletion was determined to arise by gene conversion, with the inactivated $U R A 3$ fragment on the glycosylase expression vector (see Materials and Methods) acting as a donor.

To determine how Apn1 deficiency and the location of the inserted $U R A 3$ influence the mutation spectrum of Apn1-proficient strains, LB20 (APN1, ura3-52, and URA3::ADP1) and FY69 (APN1 and $U R A 3$ at the normal locus, chromosome $\mathrm{V}$ ), a strain unrelated to LB20a, were analyzed. For the CDG- and TDG-induced mutations in LB20, the 290-bp deletion accounted for $\approx 52 \%$ and $\approx 44 \%$, respectively, of the total ura3 mutations, similar to the results for apn $1-\Delta 1$ strains; other mutation types accounted for $\approx 2 \%$ of $C D G$ mutants and $7 \%$ of TDG mutants. Thus, the near-constant proportion of the 290-bp deletion mutations in the LB strains was not likely to skew the point-mutation spectrum significantly. The mutations in the FY69 background showed a lower proportion of the 290-bp deletion ( $\approx 13 \%$ of total mutations with the ODG plasmid and $15 \%$ with CDG and TDG). The other mutation types constituted $\approx 15 \%$ of the total for FY 69 with ODG, $\approx 7 \%$ with CDG, and $\approx 9 \%$ with TDG.

Spectrum of Single-Base-Pair Substitutions. Full-length (nondeletion) PCR products of ura3 mutants were sequenced to determine the single-base-pair substitutions (Table 3). Most striking from this analysis was the observation that the point mutations generated by CDG were targeted almost exclusively to $\mathrm{G} \cdot \mathrm{C}$ base pairs, whereas the TDG-induced mutations occurred nearly exclusively at A.T base pairs. Thus, the AP sites generated by the mutant DNA glycosylases resulted in mutations specifically targeted to the base pairs recognized by CDG and TDG. This base-pair-targeting effect was observed with both replication orientations of $U R A 3$ (Table 3). In contrast, in the ODG strains (lacking CDG or TDG), mutations 
Table 3. Base substitution frequencies

\begin{tabular}{|c|c|c|c|c|c|c|c|c|}
\hline \multirow[b]{2}{*}{ Strain } & \multicolumn{3}{|c|}{ Transitions } & \multicolumn{5}{|c|}{ Transversions } \\
\hline & Total & $\mathrm{AT}>\mathrm{GC}$ & $\mathrm{GC}>\mathrm{AT}$ & Total & $\mathrm{AT}>\mathrm{TA}$ & $A T>C G$ & $\mathrm{GC}>\mathrm{TA}$ & $\mathrm{GC}>\mathrm{CG}$ \\
\hline LB20a ODG & 12 & $4(5)$ & $8(9)$ & 73 & $8(9)$ & $18(22)$ & $40(47)$ & $7(8)$ \\
\hline LB21a ODG & 7 & $2(4.5)$ & $5(11)$ & 37 & $3(7)$ & $7(16)$ & $25(57)$ & $2(4.5)$ \\
\hline LB20a CDG & 57 & $1(0.5)$ & $56(27)$ & 154 & $3(1.5)$ & $6(3)$ & 41 (19) & $104(49)$ \\
\hline LB21a CDG & 24 & 0 & $24(26)$ & 67 & 0 & $5(6)$ & $18(20)$ & $44(48)$ \\
\hline LB20 CDG & 10 & 0 & $10(24)$ & 31 & 0 & $6(15)$ & $18(44)$ & $7(17)$ \\
\hline LB20a TDG & 8 & $6(3)$ & $2(1)$ & 231 & $36(15)$ & $178(74)$ & $16(7)$ & $1(<1)$ \\
\hline LB21a TDG & 7 & $4(3)$ & $3(2)$ & 135 & $20(14)$ & $105(74)$ & $8(6)$ & $2(1)$ \\
\hline LB20 TDG & 4 & 0 & $4(9)$ & 40 & $5(11)$ & $17(39)$ & $17(39)$ & $1(2)$ \\
\hline
\end{tabular}

Data in parentheses are percentages.

were distributed at both $\mathrm{A} \cdot \mathrm{T}$ and $\mathrm{G} \cdot \mathrm{C}$ base pairs, with some preference for the latter (Table 3 ). When the CDG enzyme was expressed in the apn $1-\Delta 1$ background, predominantly $\mathrm{GC}>\mathrm{CG}$ transversions were observed, $\approx 50 \%$ of all single-base-pair substitutions. Also observed at high frequency with $\mathrm{CDG}$ were $\mathrm{GC}>\mathrm{AT}$ transitions $(\approx 26 \%)$, and GC $>$ TA transversions $(\approx 20 \%)$. Expression of the TDG enzyme in the apn $1-\Delta l$ background showed mostly AT $>$ CG $(74 \%)$ and AT $>$ TA $(\approx 15 \%)$ transversions (Table 3$)$.

CDG-induced mutants in the $A P N 1$ strain LB20 yielded a similar frequency of GC $>$ AT transitions as in the apnl- $\Delta 1$ background (24\% vs. $26-27 \%$ ) but different frequencies of GC $>$ TA transversions $(\approx 44 \%$ vs. $19-20 \%)$ and GC $>$ CG transversions $(\approx 17 \%$ vs. 48-49\%) (Table 3). In the TDG-induced mutants, AT $>$ CG transversions made up $\approx 39 \%$ of observed mutations in the $A P N 1$ strain, compared with $\approx 74 \%$ in the apn $1-\Delta 1$ background, and AT $>$ TA transversions made up $\approx 11 \%$ of observed mutations, similar to the $\approx 15 \%$ in the apn $1-\Delta 1$ strains (Table 3 ).

An $A P N 1$ strain (FY69) with $U R A 3$ at the normal chromosomal locus was also studied to determine how the location of $U R A 3$ might influence the overall spectrum of mutations. CDG-induced mutations in FY69 had similar proportions compared with LB20 of GC $>$ AT transitions (16\% vs. 24\%) and GC $>$ TA transversions $(36 \%$ vs. $44 \%)$ but a higher proportion of $\mathrm{GC}>\mathrm{CG}$ transversions (39\% vs. $19 \% ; z=2.30$ and $P<0.05$ ).

Mutations in APN1 strains expressing the TDG enzyme did not show the same degree of targeting as found for expression of CDG in these strains or of TDG expressed in the apn1- $\Delta 1$ strains. However, the overall TDG-induced mutation patterns in FY69 (data not shown) and LB20 (Table 3) were quite similar, indicating that there are no differences in the local environment between $U R A 3$ and $A D P 1:: U R A 3$ that significantly influence the mutagenic processing of T-derived AP sites.

To determine which classes of mutations were most influenced by the mutant glycosylases, we calculated how the rate of each type of single-base-pair substitution changed with expression of each glycosylase. Mutation rates were determined by normalizing the frequency of each mutation type as a function of the overall mutation rate for each strain. The overall patterns of distribution of the normalized mutation rates within each strain showed some differences between the LB20a orientation and the LB21a orientation. Both $\mathrm{GC}>\mathrm{CG}$ and $\mathrm{GC}>\mathrm{TA}$ transversions driven by $\mathrm{CDG}$ occurred at approximately three times the rate in LB20a (Fig. $2 A$ ) compared with LB21a (Fig. 2B). CDG-driven GC $>$ AT transitions were also higher in LB20a compared with LB21a (Fig. 2). In contrast, there was much less replication-orientation difference for the TDG-driven mutations (Fig. 2), especially when corrected for an AT $>$ CG hotspot (see below and see Fig. 3).

The physical distribution of mutations in the URA3 gene is shown in Fig. 3. The LB20a and LB21a orientations are directly compared in each strain, with the central axis line representing the entire $U R A 3$ sequence. For ODG, spontaneous mutations (Fig. $3 A$ ) were found somewhat more frequently in the first half of the $U R A 3$ gene. The CDG-induced mutations (Fig. $3 B$ ) were scattered a little more evenly throughout the gene. For CDG-driven mutagenesis, there were a few positions frequently mutated in both $U R A 3$ orientations at base pairs 22, 89, 181, 286, 344, 461, and 550. TDG-induced mutations in the apn1- $\triangle 1$, URA3::ADP1 background (Fig. $3 C$ ) showed a generally similar pattern for both orientations, including a pronounced $\mathrm{AT}>\mathrm{CG}$ transversion hotspot at position 449. In contrast, a TDG-generated hotspot at position 398 in LB20a was much less pronounced in the LB21a orientation (Fig. 3C). TDG expression also generated a relatively high frequency of $\mathrm{AT}>\mathrm{CG}$ mutations at position 449 in the $A P N 1^{+}$strains LB20 and FY69 (data not shown). The sequence around this hotspot (CTTTTGATGTTAGCAGAATTG; the italicized T represents the hotspot at position 449) does not provide an obvious explanation for the targeting of mutations at this position.

Effect of Gene Orientation on Distribution of Single-Base-Pair Substitutions. To determine whether replication polarity influences APsite mutagenesis, we examined the types of base substitutions in each strand of both orientations of the URA3 target (see Materials and Methods). Overall, in both LB20a and LB21a cells, the replication orientation had rather little effect. However, in both orien-
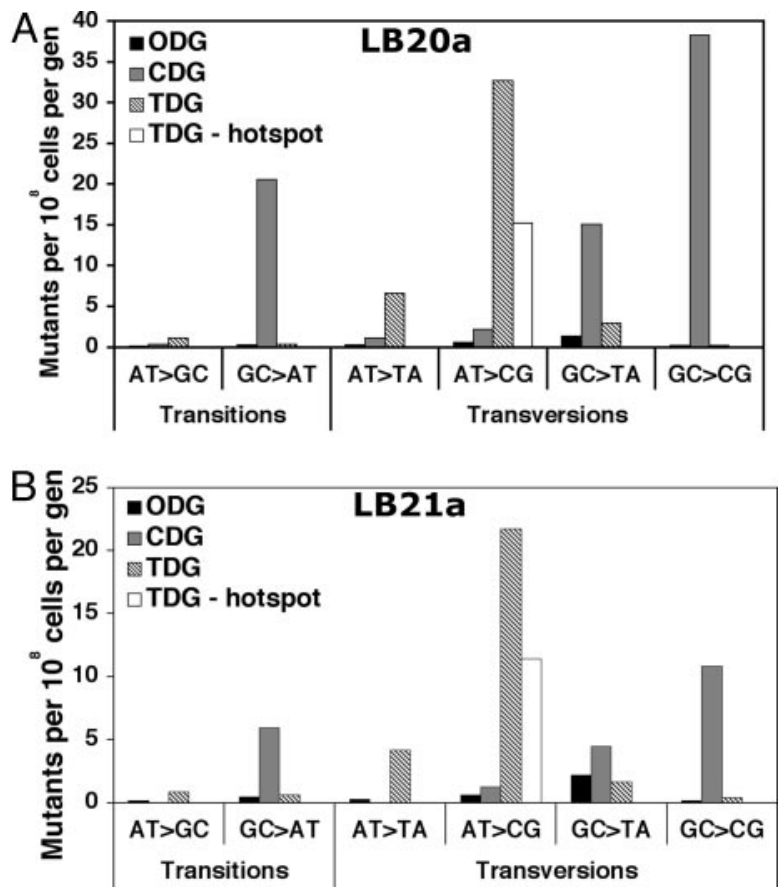

Fig. 2. Rates of individual base-substitution mutations. The data from Tables 2 and 3 were used with the overall mutation rates (Fig. 1) to estimate mutation-specific values. For the AT $>$ CG column, a value is also shown with the AT $>$ CG hotspot at position 449 (see Fig. 3C) subtracted. 

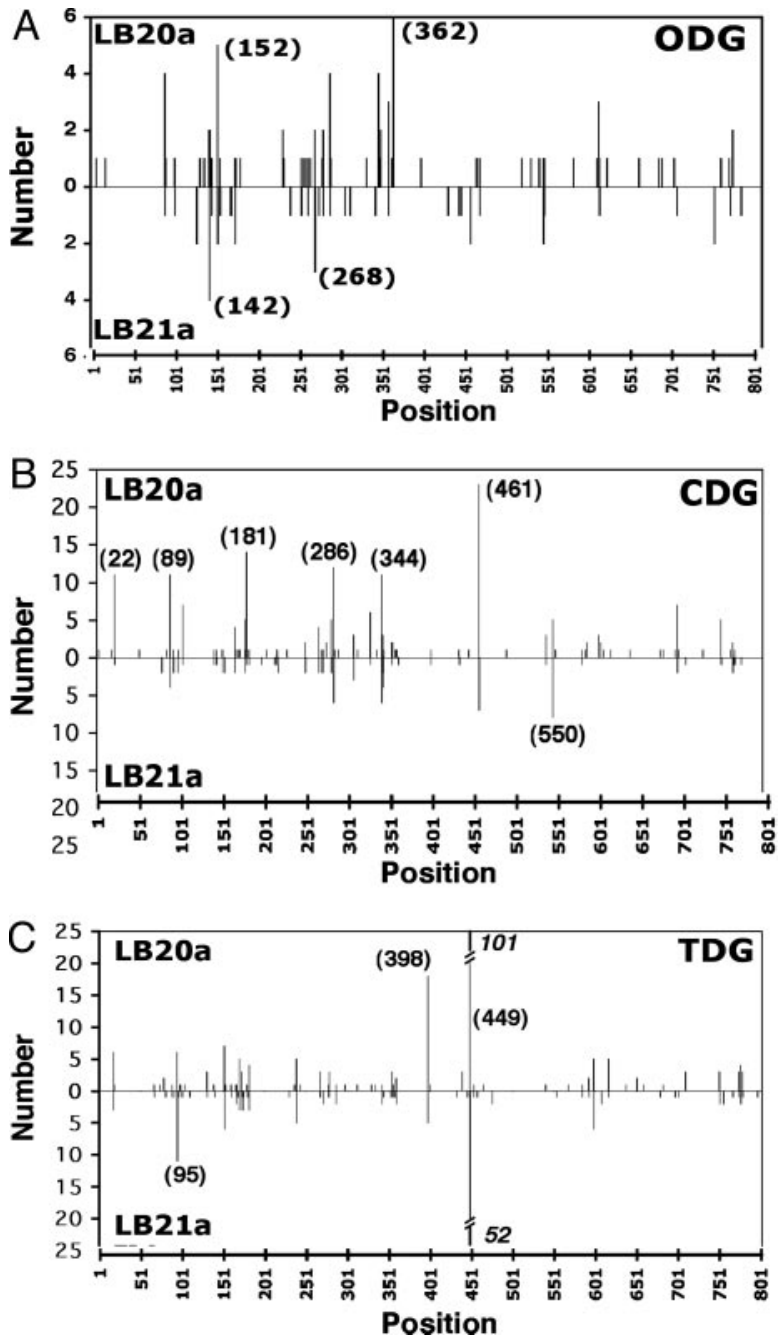

Fig. 3. Physical distribution of single-base-pair substitutions in the URA3 gene. The positions of some of the most frequent mutation sites are numbered. (A) Strains (apn1- $\Delta 1$ ) with the empty vector pODG. (B) Strains with the pCDG vector. (C) Strains with the pTDG vector.

tations, $\approx 70 \%$ of substitutions occurred in the nontranscribed strand. A $\chi^{2}$ analysis of the distribution of mutations in the transcribed vs. nontranscribed strand gave $P<0.0001$ for both LB20a and LB21a. Analysis of the URA3 sequence was performed to determine if there was an inherent bias between the strands for detectable base-pair substitutions between the strands (those giving a change in the amino acid sequence). In the nontranscribed strand, $78 \%$ of thymines and $86 \%$ of cytosines are in detectable positions, and in the transcribed strand, $86 \%$ of thymines and $95 \%$ of cytosines would be detectable. Therefore, the strand bias observed cannot be due to the influence of the sequence alone.

Further analysis showed that the observed strand bias arose almost exclusively from the TDG-driven mutations (Table 4). The CDG-driven mutations were distributed about equally between the two strands in both replication orientations. In contrast, $91 \%$ of the TDG-induced substitutions in LB20a cells occurred in the nontranscribed strand, about half of these at a single hotspot (base pair 49 ), and $9 \%$ in the transcribed strand $\left(\chi^{2}, P<0.0001\right)$. In LB21a cells, $89 \%$ occurred in the nontranscribed strand (again, about half at the base-pair-449 hotspot) and $11 \%$ in the transcribed strand $\left(\chi^{2}, P<0.0001\right)$. Clearly, the replication orientation had little effect in this system, whereas transcriptional polarity may influence TDG-driven mutagenesis quite dramatically.

The TDG-induced substitutions were then further analyzed by class (Table 4). Although AT $>$ GC transitions occurred infrequently, they were observed to originate only from damage in the nontranscribed strand. The most frequent TDG-induced mutations, $\mathrm{AT}>\mathrm{CG}$ transversions, were registered 165 times in the nontranscribed strand against only 13 times in the transcribed strand in LB20a $\left(\chi^{2}, P<0.0001\right)$ and 99 times in the nontranscribed strand vs. 6 times in the transcribed strand in LB21a $\left(\chi^{2}, P<\right.$ 0.0001). The other most frequent TDG-induced mutation, AT $>$ TA, occurred 29 times in the nontranscribed strand against only 7 times in the transcribed strand in LB20a $\left(\chi^{2}, P=0.0002\right)$. The numbers for AT> TA mutations in LB21a cells were too low to permit a strong statistical analysis.

\section{Discussion}

To analyze the mutational effects of AP sites, we have developed a system that avoids problems associated with previous approaches, such as uncertainty about what strand contained the mutagenic lesion or even its identity as an AP site. A major advantage here has been the choice of altered human UDG enzymes that selectively remove either cytosines or thymines to generate regular AP sites (16). The use of plasmids with site-specific AP lesions $(9,13,34,35)$ also allows specificity concerning the location of the sites, but it is uncertain whether the incoming DNA molecules are repaired and replicated identically to chromosomal DNA. Installing the $U R A 3$ mutation target near ARS309 in our system avoids this question and, with the two orientations, provides known replication polarity (36).

Our results show several important effects. The expected targeting of the engineered CDG and TDG enzymes based on in vitro assays (16) is confirmed in vivo by their nearly complete biases for mutations at $\mathrm{G} \cdot \mathrm{C}$ and $\mathrm{A} \cdot \mathrm{T}$ base pairs, respectively (Table 3 ). This degree of targeting was much greater than that observed for mutagenesis by TDG and CDG expression in AP-endonucleasedeficient $E$. coli (33). The observed base-pair substitutions were overwhelmingly transversions, indicating that the major mutagenic pathways for AP sites in S. cerevisiae involve the insertion of pyrimidines opposite the abasic residue. That dCMP was the nucleotide most frequently inserted, with dAMP the next-most-

Table 4. Strand-specific mutation frequencies

\begin{tabular}{|c|c|c|c|c|c|c|c|c|}
\hline \multirow[b]{2}{*}{ Mutations } & \multicolumn{8}{|c|}{ Strain } \\
\hline & \multicolumn{2}{|c|}{ LB20a CDG } & \multicolumn{2}{|c|}{ LB21a CDG } & \multicolumn{2}{|c|}{ LB20a TDG } & \multicolumn{2}{|c|}{ LB21a TDG } \\
\hline Total & 201 & & 86 & & 220 & & 129 & \\
\hline Nontranscribed & 108 & $54 \%$ & 35 & $41 \%$ & 200 & $91 \%$ & 115 & $89 \%$ \\
\hline Transcribed & 93 & $46 \%$ & 51 & $59 \%$ & 20 & $9 \%$ & 14 & $11 \%$ \\
\hline Transitions & & & & & Non-Tx & Tx & Non-Tx & Tx \\
\hline$A T>G C$ & & & & & 6 & 0 & 4 & 0 \\
\hline \multicolumn{9}{|l|}{ Transversions } \\
\hline $\mathrm{AT}>\mathrm{CG}$ & & & & & 165 & 13 & 99 & 6 \\
\hline $\mathrm{At}>\mathrm{TA}$ & & & & & 29 & 7 & 12 & 8 \\
\hline
\end{tabular}

Non-Tx, nontranscribed; Tx, transcribed. Percentages represent the percent of total frequency. 
frequent, is consistent with previous studies in $S$. cerevisiae $(9,13$, $14,37)$, confirming results obtained from distinctly different experimental methods. Insertion of dGMP occurred rarely in all studies where it could be established unambiguously.

The most unusual category of point mutations provoked by AP sites are GC>TA and AT $>$ TA transversions seen in response to CDG and TDG, respectively (Table 3 ). Such mutations could arise from insertion of dTMP opposite the AP site during mutagenic DNA synthesis, an activity that has not been associated with yeast DNA polymerases. There is evidence that Rev1 modulates dTMP insertion independently of its dCMP transferase activity (14), which may indicate that another enzyme or modifying activity is yet to be found. Alternatively, some aspect of DNA or chromosome structure in vivo allows dTMP insertion that has not been observed in vitro. Analysis of the genetic dependence of specific mutation subtypes should shed some light on this intriguing observation.

Our results with the Rev1- and Pol- $\zeta$-deficient strains showed several informative effects. The rev1s allele lowered the basal mutation rate 13 -fold relative to the $R E V^{+}$strain (Table 1), consistent with published observations (17). Although CDG and TDG elevated this rate $\approx 8$-fold and 3 -fold, respectively, the rates were still far below those observed in $R E V^{+}$cells expressing the glycosylases, and the apparent increases in mutation rates were not statistically significant. Thus, mutagenesis by AP sites in yeast depends heavily on $R E V 1$, but there is still a small REV1independent component. The residual CDG-induced mutagenesis in the rev $1 \Delta$ strain could include the GC $>$ AT A-rule substitutions, because this class of mutation is the most frequent in Rev1-deficient strains (38), but such a simple picture would predict, based on Table 3 , a higher CDG-induced mutation rate than we observed. The CDG-induced GC $>$ TA and TDG-induced AT $>$ TA transversions could also be formed in a Rev1-independent fashion but, again, would seem to be making a rather minor contribution in the rev1s strain. In the case of the rev $7 \Delta$ strain, mutagenesis in response to CDG or TDG was virtually completely suppressed (values not significantly different from ODG), although there may be a slight mutagenic trend in response to TDG (Table 1). However, Rev7deficiency did not have nearly the effect on the basal mutation rate as did Rev1-deficiency. Surprisingly, the 290-bp deletions produced by gene conversion evidently also strongly depend on REV1 and $R E V 7$. Because gene conversions constituted $\approx 40 \%$ of the total mutations in LB20a (Table 2), one would expect a much higher residual mutation rate in rev $1 \Delta$ and $r e v 7 \Delta$ expressing CDG or TDG, if the gene-conversion events were independent of the TLS en-

1. Lindahl, T. (1993) Nature 362, 709-715.

2. Loeb, L. A. \& Preston, B. D. (1986) Annu. Rev Genet. 20, 201-230.

3. Prakash, S., Johnson, R. E. \& Prakash, L. (2004) Annu. Rev. Biochem.

4. Nelson, J. R., Lawrence, C. W. \& Hinkle, D. C. (1996) Nature 382, 729-731.

5. Haracska, L., Unk, I., Johnson, R. E., Johansson, E., Burgers, P. M., Prakash, S. \& Prakash, L. (2001) Genes Dev. 15, 945-954.

6. Huang, M. E., Rio, A. G., Galibert, M. D. \& Galibert, F. (2002) Genetics 160, 1409-1422

7. Haracska, L Washington, M. T. Prakash, S. \& Prakash, L (2001) J Biol Chem 276, 6861-6866.

8. Zhao, B., Xie, Z., Shen, H. \& Wang, Z. (2004) Nucleic Acids Res. 32, 3984-3994

9. Gibbs, P. E., McDonald, J., Woodgate, R. \& Lawrence, C. W. (2005) Genetics 169, 575-582.

10. Lehmann, A. R. (2002) Mutat. Res. 509, 23-34.

11. Jansen, J. G. \& de Wind, N. (2003) DNA Repair 2, 1075-1085.

12. Kunz, B. A., Henson, E. S., Roche, H., Ramotar, D., Nunoshiba, T. \& Demple, B. (1994) Proc. Natl. Acad. Sci. USA 91, 8165-8169.

13. Gibbs, P. E. \& Lawrence, C. W. (1995) J. Mol. Biol. 251, 229-236.

14. Otsuka, C., Sanadai, S., Hata, Y., Okuto, H., Noskov, V. N., Loakes, D. \& Negishi, K. (2002) Nucleic Acids Res. 30, 5129-5135.

15. Guillet, M. \& Boiteux, S. (2003) Mol. Cell. Biol. 23, 8386-8394.

16. Kavli, B., Slupphaug, G., Mol, C. D., Arvai, A. S., Peterson, S. B., Tainer, J. A. \& Krokan, H. E. (1996) EMBO J. 15, 3442-3447.

17. Glassner, B. J., Rasmussen, L. J., Najarian, M. T., Posnick, L. M. \& Samson, L. D. (1998) Proc. Natl. Acad. Sci. USA 95, 9997-10002.

18. Alani, E., Cao, L. \& Kleckner, N. (1987) Genetics 116, 541-545.

19. Ramotar, D., Popoff, S. C., Gralla, E. B. \& Demple, B. (1991) Mol. Cell. Biol. 11, 4537-4544

20. Purnelle, B., Skala, J. \& Goffeau, A. (1991) Yeast 7, 867-872.

21. Skala, J., Purnelle, B. \& Goffeau, A. (1992) Yeast 8, 409-417.

22. Lemontt, J. F. (1971) Genetics 68, 21-33.

23. Ausubel, F. M. Bent, R., Kingston, R. E., Moore, D. D., Seidman, J. G., Smith, J. A., Struhl, K., Albright, L. M., Coen, D. M. \& Varki, A. (1996) Current Protocols In Molecular Biology, ed. Janssen, K. (Wiley, New York), 13.1.1-13.1.7.

24. Hoffman, G. A., Garrison, T. R. \& Dohlman, H. G. (2002) Methods Enzymol. 344, 617-631. zymes. The mechanism of the apparent TLS-dependence of gene conversion induced by endogenous AP-site formation is unclear, but will be interesting to explore.

We were surprised to find only modest effects of the replication orientation on mutation rates or specificity in the URA3 reporter gene (Fig. 1; Table 4). Others have reported strand specificity for spontaneous mutations that appear to be due to polymerase errors $(39,40)$. Evidently, the processes in operation at AP lesions in yeast differ from those that give rise to strand-specificity in replication errors. Whereas, in E. coli, replication polarity affects replicative $(41,42)$ and damaged-induced (43) mutagenesis differently, the situation in yeast has been unresolved, and our results shed light on one type of DNA damage.

There was, however, a very strong bias for TDG-induced mutations that depended on the transcriptional orientation (Table 4). TDG-induced transversions were nearly 10 -fold more frequent for the nontranscribed than for the transcribed strand. Such a difference could arise from an unknown Apn1-independent repair mechanism that operates preferentially on the transcribed strand, for example. However, such a bias was not observed for CDGdriven mutations, which suggests transcribed-strand-specific processing of AP sites opposite A, or discrimination in the TDG glycosylase itself. In the absence of Apn1, AP sites could become more susceptible to AP lyases, such as Ntg1 and Ogg1. However, these enzymes cleave AP sites preferentially opposite pyrimidines (44) or opposite C nearly exclusively (45), so their involvement would seem to require $\mathrm{AP} / \mathrm{C}$ and $\mathrm{AP} / \mathrm{T}$ sites generated by replication past the AP site. The mutator glycosylase properties also do not provide a ready explanation: TDG is about equally effective on single-stranded compared with double-stranded DNA, whereas CDG has some preference for single-stranded DNA substrates (16).

A refined analysis of AP site mutagenesis supports the proposed critical roles of some TLS proteins, notably Rev1 and Rev7. Some mutation types, and the unexpected effects of transcription polarity, indicate that significant mutational pathways remain to be analyzed. With such effects detectable, even with the transient expression of the mutator DNA glycosylases, it will be of interest to know whether more severe or chronic accumulation of abasic DNA damage changes the contributions of novel mutational pathways.

We thank Graham Walker for helpful discussions and members of the Demple laboratory for advice and discussions. This work was supported by National Institutes of Health Grant GM40000 (to B.D.), by funds from the European Union (to H.E.K.), and by Training Grant in Radiation Biology T32-09078 (to P.A. and R.A.O.B.).

25. Boeke, J. D., Trueheart, J., Natsoulis, G. \& Fink, G. R. (1987) Methods Enzymol. 154, 164-175.

26. Von Borstel, R. C. (1978) in Methods in Cell Biology, ed. Prescott, D. M. (Academic, New York), Vol. 20, pp. 1-24.

27. Wilson, D. M., III, Bennett, R. A. O., Marquis, J. C., Ansari, P. \& Demple, B. (1995) Nucleic Acids Res. 23, 5027-5033.

28. Clairmont, C. A. \& Sweasy, J. B. (1998) J. Bacteriol. 180, 2292-2297.

29. Johnson, R. E., Torres-Ramos, C. A., Izumi, T., Mitra, S., Prakash, S. \& Prakash, L. (1998) Genes Dev. 12, 3137-3143.

30. Bennett, R. A. O. (1999) Mol. Cell. Biol. 19, 1800-1809.

31. Nakamura, J., Walker, V. E., Upton, P. B., Chiang, S. Y., Kow, Y. W. \& Swenberg, J. A. (1998) Cancer Res. 58, 222-225.

32. Atamna, H., Cheung, I. \& Ames, B. N. (2000) Proc. Natl. Acad. Sci. USA 97, 686-691.

33. Otterlei, M., Kavli, B., Standal, R., Skjelbred, C., Bharati, S. \& Krokan, H. E. (2000) EMBO J. 19, 5542-5551.

34. Gentil, A., Cabral-Neto, J. B., Mariage-Samson, R., Margot, A., Imbach, J. L., Rayner, B. \& Sarasin, A. (1992) J. Mol. Biol. 227, 981-984.

35. Neto, J. B., Gentil, A., Cabral, R. E. \& Sarasin, A. (1992) J. Biol. Chem. 267, 19718-19723.

36. Palzkill, T. G., Oliver, S. G. \& Newlon, C. S. (1986) Nucleic Acids Res. 14, 6247-6264.

37. Otsuka, C., Loakes, D. \& Negishi, K. (2002) Nucleic Acids Res. Suppl., 87-88.

38. Nelson, J. R., Gibbs, P. E., Nowicka, A. M., Hinkle, D. C. \& Lawrence, C. W. (2000) Mol. Microbiol. 37, 549-554.

39. Pavlov, Y. I., Newlon, C. S. \& Kunkel, T. A. (2002) Mol. Cell 10, 207-213.

40. Pavlov, Y. I., Mian, I. M. \& Kunkel, T. A. (2003) Curr. Biol. 13, 744-748.

41. Fijalkowska, I. J., Jonczyk, P., Tkaczyk, M. M., Bialoskorska, M. \& Schaaper, R. M. (1998) Proc. Natl. Acad. Sci. USA 95, 10020-10025.

42. Maliszewska-Tkaczyk, M., Jonczyk, P., Bialoskorska, M., Schaaper, R. M. \& Fijalkowska, I. J. (2000) Proc. Natl. Acad. Sci. USA 97, 12678-12683.

43. Gawel, D., Maliszewska-Tkaczyk, M., Jonczyk, P., Schaaper, R. M. \& Fijalkowska, I. J. (2002) J. Bacteriol. 184, 4449-4454.

44. Meadows, K. L., Song, B. \& Doetsch, P. W. (2003) Nucleic Acids Res. 31, 5560-5567.

45. Girard, P. M., Guibourt, N. \& Boiteux, S. (1997) Nucleic Acids Res. 25, 3204-3211. 